\title{
Criminologie
}

\section{Regards croisés sur des enjeux et reconfigurations du dispositif carcéral fédéral pour les femmes au Canada}

\section{Sylvie Frigon}

Volume 35, numéro 2, automne 2002

Femmes et enfermement au Canada : une décennie de réformes

URI : https://id.erudit.org/iderudit/008287ar

DOI : https://doi.org/10.7202/008287ar

Aller au sommaire du numéro

Éditeur(s)

Les Presses de l'Université de Montréal

ISSN

0316-0041 (imprimé)

1492-1367 (numérique)

Découvrir la revue

Citer ce document

Frigon, S. (2002). Regards croisés sur des enjeux et reconfigurations du dispositif carcéral fédéral pour les femmes au Canada. Criminologie, 35(2), 3-7. https://doi.org/10.7202/008287ar d'utilisation que vous pouvez consulter en ligne. 


\section{Regards croisés sur des enjeux et reconfigurations du dispositif carcéral fédéral pour les femmes au Canada}

Sylvie Frigon

Professeure

Département de criminologie

Université d'Ottawa

sfrigon@uottawa.ca

Ce numéro thématique propose un bilan à la fois descriptif et critique, théorique et empirique, du traitement pénal réservé aux femmes au Canada depuis une décennie (1990-2000). Une décennie marquée par de profonds changements en matière d'enfermement des femmes, mais aussi, plus généralement, de justice faite aux femmes. Ce numéro innove et ce, à deux niveaux. Premièrement, dans la présentation, en plus de six articles thématiques, il comprend deux commentaires de deux spécialistes d'horizons différents et un article-bilan du Service correctionnel du Canada (SCC). Deuxièmement, ce numéro servira de tremplin à une journée d'études à l'automne 2002, parrainée par le SCC, consacrée à une réflexion sur les perspectives d'avenir en matière d'incarcération féminine.

Ici comme ailleurs, l'industrie carcérale féminine a fait l'objet de différentes manifestations, réunions et publications (voir Frigon et Faith, ce numéro). Au Canada, le traitement des femmes justiciables a également été au cœur des préoccupations correctionnelles, comme en témoignent trois principales études et/ou enquêtes. Premièrement, La création de choix, en 1990, sur les femmes incarcérées sous responsabilité fédérale a contribué à la fermeture de la prison des femmes de Kingston et à l'ouverture de cinq nouveaux centres régionaux de détention, sujet sur lequel ce présent numéro se penchera principalement. Deuxièmement, il y eut la 
Commission d'enquête sur certains événements survenus à La Prison des femmes de Kingston, présidée par la juge L. Arbour en 1996, sur la légitimité d'une intervention masculine anti-émeute en 1994. Finalement, la juge L. Ratushny mènera l'Examen de la légitime défense, portant sur les femmes homicidaires, en $1997^{1}$. Ces trois rapports ont en commun de faire ressortir les liens entre les processus de criminalisation et de victimisation des femmes, c'est-à-dire de faire ressortir les liens entre le passage à l'acte et le fait d'avoir été victime. La conférence intitulée La résistance des femmes: de la victimisation à la criminalisation, tenue à Ottawa en octobre 2001, a pris acte de cette relation. Cette conférence, organisée par l'Association canadienne des sociétés Elizabeth Fry (ACSEF) et l'Association canadienne des centres contre le viol (ACCCV), regroupait plus de 120 ateliers et groupes de travail, dont plusieurs se penchaient sur les conséquences de La création de choix et du traitement fait aux femmes en prison.

Avant 1990, les femmes en prison étaient, à toute fin pratique, presque entièrement ignorées. Elles ne constituaient pas une priorité en raison de leur petit nombre et parce qu'elles ne posaient pas de problème majeur en matière de gestion, de sécurité et d'opinion publique. D'un «inconvénient social $»^{2}$ à être «trop peu nombreuses pour compter $»^{3}$, les femmes incarcérées sont devenues un objet de préoccupation sociale et pénale grandissante. Elles constituent un cas de figure qui nous renvoie à la «centralité de la prison dans notre système pénal, en dépit de la constance de son échec» (Perrot, 2001).

Au Canada, pendant la décennie des années 1990, les femmes incarcérées représentaient environ $7 \%$ de la population carcérale provinciale (sentences de moins de deux ans) et $4 \%$ de la population carcérale fédérale (sentences de deux ans et plus). Par exemple, en 1995, 305 femmes purgeaient des peines fédérales, comparativement à 13234 hommes.

Jusqu'à très récemment, le fait qu'il n'existait qu'un seul pénitencier pour femmes au Canada posait un certain nombre de difficultés. Ces difficultés se manifestaient aussi bien à l'intérieur qu'à l'extérieur de l'institution et incluaient la disponibilité de programmes, de formations et de services, la classification des détenues, les tentatives de suicides et les suicides complétés, l'éloignement des familles et des enfants, la dislocation culturelle et

1. Pour une discussion plus détaillée de ce rapport, voir Frigon et Viau (2000).

2. Titre emprunté à L. Berzins et R. Colette-Carrière (1979). Les femmes en prison : un inconvénient social! Santé mentale au Québec, IV (2), 87-103.

3. Traduction libre du titre de l'ouvrage de E.A. Adelberg et C. Currie (1987). Too few to count: Canadian women in conflict with the law. Vancouver : Press Gang Publishers. 
linguistique et l'accès aux programmes de réinsertion sociale. Pour toutes ces raisons et bien d'autres, le groupe d'étude La création de choix recommandait la fermeture de la prison des femmes de Kingston en Ontario et l'ouverture de cinq nouveaux centres régionaux de détention dispersés à travers le Canada, afin d'atténuer, si possible, certains des coûts liés à un séjour dans un établissement carcéral.

Afin d'analyser la situation, nous allons tenter de saisir quelles sont les conséquences de ces transformations. Quels sont les gains? Quelles en sont les dérives? Dans quelle mesure ces nouvelles pratiques de gouvernance préconisées dans $L a$ création de choix débouchent-elles véritablement sur des interventions plus humaines, favorisant l'autonomisation? Mais également, quels impacts ces nouvelles pratiques de gouvernance ont-elles sur la distribution du pouvoir entre acteurs sociaux (incarcérées) et sur le contrôle pénal et l'exercice du pouvoir? Quelles sont les dynamiques sociopolitiques et institutionnelles pouvant influer sur ces pratiques? À l'aide de six articles et de deux commentaires, nous souhaitons jeter des balises et proposer des pistes de réflexion.

Sur le plan organisationnel, le numéro commence par un article de Sylvie Frigon, dans lequel la démarche de l'auteure s'organise autour de quatre objectifs spécifiques : 1) dégager un portrait des femmes purgeant une peine fédérale au Canada; 2) discuter des conditions d'émergence du groupe de travail La création de choix (1990); 3) poser un regard sur les nouveaux centres de détention et la nouvelle philosophie pénale; enfin 4) examiner les reconfigurations et les redéploiements de la surveillance et de la punition enchâssés dans la nouvelle philosophie pénale. Il est suivi par un article-bilan du Service correctionnel du Canada, signé par Marie-Andrée Cyrenne, qui offre un profil des femmes purgeant une peine fédérale, une description des nouveaux établissements, des divers programmes, de la stratégie d'intervention intensive pour incarcérées à sécurité maximale, et des programmes de mise en liberté, pour enfin se terminer sur les défis qui demeurent.

Pour leur part, Margaret Shaw et Kelly Hannah-Moffat proposent une réflexion critique en faisant un retour sur les enjeux des réformes dans la dernière décennie au sein de ce nouvel univers carcéral féminin fédéral, à l'aide de deux cas de figure qui échappent aux réformes actuelles : les femmes classées à sécurité minimale et maximale et les mesures communautaires.

Étant donné la centralité de la problématique des femmes autochtones dans la période et dans l'espace qui nous intéressent, l'article de 
Renée Brassard et de Mylène Jaccoud est fort pertinent, car les auteures décrivent l'état actuel des travaux sur l'enfermement des femmes autochtones, la façon dont la question a été problématisée jusqu'à ce jour, pour finalement proposer un modèle d'analyse reconstruisant la question de l'enfermement des femmes autochtones.

Une autre zone d'intérêt explorée par l'article suivant, signé par Brigitte Blanchard, réfere à la situation des mères incarcérées et de leurs enfants au Québec, compte tenu de la mise en place de différents programmes mère-enfant tant dans les juridictions fédérale que provinciale. À l'aide de la collaboration d'une centaine de mères et des réponses pour plus de deux cents de leurs enfants, l'auteure décrit le profil des mères, le profil des enfants et de leur milieu de vie, des liens entretenus entre les mères et les enfants avant, pendant et après l'incarcération et des programmes mis sur pied pour faciliter le maintien de ces liens. Elle constate que malgré la mise sur pied de programmes novateurs, la situation est fort complexe et mérite d'être étudiée plus sérieusement.

Dans un essai engagé intitulé La résistance à la pénalité : un impératif féministe, Karlene Faith entreprend une remise en question en profondeur de la légitimité de la prison, de l'essor fulgurant de l'arsenal carcéral, du maintien de la logique de punition au cœur même de ces soidisant nouvelles philosophie et gestion pénale. En reprenant l'histoire de l'incarcération des femmes et de ses réformes, l'auteure revendique la fin de la prison au nom de la justice sociale et du féminisme.

Deux commentaires clôturent cette partie du numéro. Toujours dans l'esprit d'effectuer un bilan critique de la situation des femmes incarcérées au Canada, deux auteures de deux univers différents partagent leurs inquiétudes sur la situation des femmes incarcérées. D'une part, MarieAndrée Bertrand, dans son commentaire intitulé Progrès, recul et stagnation. Tableau contrasté des conditions de vie des femmes incarcérées au Canada, se demande comment penser l'incarcération des femmes, lorsque les personnes en cause, la durée de leur peine et leur niveau de sécurité sont impossibles à saisir, car les «statistiques criminelles canadiennes parlent de personnes sans sexe et sans identité ethnique ou culturelle». D'autre part, dans un commentaire à la fois très passionné et documenté, Kim Pate, directrice des Sociétés Elizabeth Fry du Canada, dresse un bilan très sombre de la situation actuelle, malgré les efforts déployés dans la dernière décennie. Elle sonne l'alarme sur l'augmentation du recours à l'incarcération pour les femmes, sur l'accroissement du filet pénal, sur les décalages entre la vision enchâssée dans La création de choix et l'implan- 
tation des réformes, sur la mise à l'écart de certaines catégories de femmes et sur les violations des droits et libertés.

Il reste beaucoup à dire, à faire, à penser, à déconstruire et à réinventer. Nous n'avons pas tout dit. Plusieurs zones d'ombre demeurent. Malgré les nouveautés et l'actualité des réaménagements de la justice, de la philosophie pénale, des institutions et des dispositifs, la logique carcérale reste intacte et permet, de surcroît, de renouveler sa légitimité et son efficacité. En effet, les nouvelles reconfigurations du pénal reconduisent aux impératifs et aux limites liés à cette logique. Les auteures, dans ce numéro, réclament un nouveau dialogue pour repenser l'incarcération des femmes. Nous espérons modestement avoir balisé de futurs échanges et déblayé le terrain.

\section{Références}

Adelberg, E.A., \& Currie, C. (1987). Too few to count: Canadian women in conflict with the law. Vancouver : Press Gang Publishers.

Arbour, L. (1996). Commission d'enquête sur certains événements survenus à la Prison des femmes de Kingston. Ottawa : Ministère des Approvisionnements et Services Canada.

Berzins, L., \& Colette-Carrière, R. (1979). Les femmes en prison : un inconvénient social! Santé mentale au Québec, IV (2), 87-103.

Frigon, S., \& Viau, L. (2000). Les femmes condamnées pour homicide et l'Examen de la légitime défense (rapport Ratushny): portée juridique et sociale. Criminologie, 33 (1), 97-119.

Perrot, M. (2001). Les ombres de l'histoire : crime et châtiment au XIX'. Paris : Flammarion.

Ratushny, L. (1997). Self-defence review, final report - Examen de la légitime défense, rapport final. Ottawa : Rapport soumis au ministre de la Justice du Canada et au Solliciteur général du Canada.

Service correctionnel du Canada (1990). La création de choix : rapport du groupe d'étude sur les femmes purgeant une peine fédérale. Ottawa : Ministère des Approvisionnements et Services. 